and A. Kimura. 1985. A novel method for transformation of intact yeast cells by electroinjection of plasmid DNA. Appl. Microbiol. Biotechnol. 21:336-339.

4.Ito, H., Y. Fukuda, K. Murata, and A. Kimura. 1983. Transformation of intact yeast cells treated with alkali cations. J. Bacteriol. 153:163-168.

5.Schiestl, R.H. and R.D. Gietz. 1989. High efficiency transformation of intact yeast cells using single stranded nucleic acids as a carrier. Curr. Genet. 16:339-346.

6.Burke, D.T., G.F. Carle, and M.V. Olson. 1987. Cloning of large segments of exogenous DNA into yeast by means of artificial chromosome vectors. Science 236:806-812.

7.Vollrath, D., R.W. Davis, C. Connelly, and P. Hieter. 1988. Physical mapping of large DNA by chromosome fragmentation. Proc. Natl. Acad. Sci. USA 85:6027-6031.

8.Widianto, D., Y. Mukai, K. Kim, S. Harashima, and Y. Oshima. 1996. One-step splitting of a chromosome in haploid cells of Saccharomyces cerevisiae and its effect on the cell proliferation. J. Ferm. Bioeng. 82:199-204.

9.Bauchwitz, R. and F. Costantini. 1998. YAC transgenesis: a study of conditions to protect YAC DNA from breakage and a protocol for transfection. Biochim. Biophys. Acta 1401: 21-37.

10.Connelly, C., M.K. McCormick, J. Shero, and P. Hieter. 1991. Polyamines eliminate an extreme size bias against transformation of large yeast artificial chromosome DNA. Genomics 10:10-16.

11.Sone, T., E. Nagamori, T. Ikeuchi, A. Mizukami, Y. Takakura, S. Kajiyama, E. Fukusaki, S. Harashima, et al. 2002. A novel gene delivery system in plants with calcium alginate micro-beads. J. Biosci. Bioeng. 94:87-91.

12.Widianto, D., E. Yamamoto, M. Sugiyama, Y. Mukai, Y. Kaneko, Y. Oshima, M. Nishizawa, and S. Harashima. 2003. Creating a Saccharomyces cerevisiae haploid strain with 21 chromosomes. J. Biosci. Bioeng. 95:89-94.

13.Gerring, S.L., C. Connelly, and P. Hieter. 1991. Positional mapping of genes by chromosome blotting and chromosome fragmentation. Methods Enzymol. 194:57-77.

14.McCormick, M.K., J. Shero, C. Connelly, S. Antonarakis, and P. Hieter. 1990. Methods for cloning large DNA segments as artificial chromosomes in S. cerevisiae. Techniques 2:65-71.

15.Burgers, P.M. and K.J. Percival. 1987. Transformation of yeast spheroplasts without cell fusion. Anal. Biochem. 163:391-397.

16.Luo, D. and W.M. Saltzman. 2000. Enhancement of transfection by physical concentration of DNA at the cell surface. Nat. Biotechnol. 18: 893-895.

Received 11 February 2003; accepted 12 June 2003.

Address correspondence to Kiichi Fukui, Department of Biotechnology, Graduate School of Engineering, Osaka University, 2-1 Yamadaoka, Suita, Osaka 565-0871, Japan.e-mail:kfukui@bio.eng.osaka-u.ac.jp

\title{
Selection of candidate housekeeping controls in tomato plants using EST data
}

\author{
Jeffrey S. Coker and Eric Davies \\ North Carolina State University, Raleigh, NC, USA
}

BioTechniques 35:740-748 (October 2003)

Because the expression levels of housekeeping genes are relatively constant in most tissues, they are often useful controls when quantifying gene expression. We present an analytical method for identifying candidate housekeeping controls using expressed sequence tags (ESTs) from The Institute for Genomic Research Tomato Gene Index. We found relative expression levels for a collection of 127 transcripts and calculated the percentage of cDNA libraries that had expression levels (for a given transcript) within 2-fold through 10-fold ranges. When all libraries were considered together, the highest ranked housekeeping controls included transcripts for a DnaJ-like protein, translationally controlled tumor protein, two $\alpha$-tubulins, cyclophilin, and glyceraldehyde-3-phosphate dehydrogenase (GAPDH). For each organ (leaf, root, fruit, and flower), at least one transcript was found that occurred within a 2-fold range of expression in all respective libraries. These included transcripts for $\alpha$-tubulin, DnaJ-like proteins, phosphoglycerate kinase, and GAPDH, although different transcripts appear better suited than others for different tissues. This analytical method is useful for identifying candidate housekeeping controls in particular tissues and at particular levels of expression and would be relevant for any species for which significant EST data exist.

\section{INTRODUCTION}

Expressed sequence tags (ESTs) are partial, single-pass sequences from randomly selected cDNA clones. ESTs in public databases are a useful tool for identifying novel genes and assembling contigs $(1,2)$, as well as for preliminary analysis of gene expression $(3,4)$. Previous studies have investigated methods of detecting significant differences in the relative expression levels of particular genes using EST databases. For example, statistical tests have been devised to compare the abundance of individual genes between two cDNA libraries (5) and between any number of libraries $(6,7)$. These methods focus on detecting genes that are up-regulated or down-regulated, and not on identifying genes whose relative expression levels are constant. The current study demonstrates a method to address the latter.

Housekeeping genes encode proteins that typically function in basic cell metabolism/upkeep. Because the expression levels of housekeeping genes are relatively constant in most tissues, they are often useful controls when quantifying gene expression. Housekeeping controls allow the consistency of tissue collection, mRNA extraction, and other procedures to be verified, thus supporting conclusions about differentially expressed genes. They also help to show what levels of variation are to be expected in particular experiments and, therefore, how to distinguish differentially expressed genes from those oscillating due to random chance (8). Even though control transcripts have been used for the last quarter century, they have inherent limitations $(9,10)$. Suzuki et al. (11) reviewed the pitfalls of the two most popular controls in mammals, glyceraldehyde-3-phosphate dehydrogenase (GAPDH) and actin, while Lee et al. (12) observed that 12 housekeeping genes (GAPDH, actin, phosphoglycerate kinase, tubulin, etc.) typically used as controls show considerable variability in microarray data sets.

In the current study, we investigated whether or not a database of tomato ESTs supports the use of housekeeping control genes and then ranked candidate control genes in leaves, roots, fruits, and flowers. 


\section{MATERIALS AND METHODS}

\section{Data Mining}

The recent releases of EST projects for tomato (13), Arabidopsis thaliana $(14,15)$, Medicago trunculata (16), and potato (17) allow the analysis of relative expression levels in various plant tissues (4). The TIGR TGI (The Institute for Genomic Research Tomato Gene Index, version 9.0; http: //www.tigr.org/tdb/tgi/lgi/) database contains 156,645 ESTs, which have been clustered into a set of 15,925 tentative consensus sequences $(13,18)$. We used tomato cDNA libraries in the TIGR TGI with greater than 500 total ESTs to mine EST frequencies for individual transcripts.

Tomato genes in our analysis included homologs of the most commonly used housekeeping genes $(9,12)$, homologs of a set of the most abundant ESTs found in 9 available potato libraries (17), and a selection of translationrelated genes, since they often have abundant transcripts (9). Table 1 contains a summary of the 127 tentative consensus sequences (TCs) that were

Table 1. Summary of Tentative Consensus Sequences (TCs) from the TIGR TGI that Were

Analyzed for Their Potential as Housekeeping Control Genes

\begin{tabular}{lc} 
Putative gene & No.TCs \\
\hline Actin & 15 \\
$\alpha$-Tubulin & 2 \\
Annexin p34 & 1 \\
AT5g28840/F7P1_20 (unknown protein) & 2 \\
Catalase & 3 \\
Cyclophilin & 5 \\
Cys protease & 5 \\
DnaJ-like protein & 20 \\
Glucose-6-phosphate dehydrogenase & 4 \\
Glutamate dehydrogenase & 2 \\
Glyceraldehyde 3-phosphate dehydrogenase (GAPDH) & 11 \\
Heat shock cognate protein 80 & 4 \\
Lactate dehydrogenase & 2 \\
Mitochondrial formate dehydrogenase precursor & 2 \\
Phospho-2-dehydro-3-deoxyheptonate aldolase & 3 \\
Phosphoglycerate kinase & 2 \\
Phosphoglycerate mutase & 1 \\
Ribosomal proteins & 12 \\
S-adenosylmethionine decarboxylase (SAMDC) & 3 \\
Translation elongation factors (1 and 2) & 4 \\
Translation initiation factor & 4 \\
Translationally controlled tumor protein (TCTP; P23) & 2 \\
Ubiquitin & 17 \\
UDP-glucose: protein transglucosylase & 1 \\
Total & 127 \\
aThe 12 ribosomal proteins included S17, S25, L17, L37, L38, L30, \\
L2 (2 of them), L19 (2 of them), L3, and 60S acidic. & \\
& \\
&
\end{tabular}

examined, each of which represents an individual, putative cDNA.

\section{Calculation of Relative Expression Levels}

Relative expression levels were calculated by dividing the number of ESTs representing a given cDNA by the total number of ESTs in a given cDNA library, and then multiplying by 1000 . For example, one tomato transcript for actin (TIGR TGI acc. no. TC116116) was represented by 4 ESTs (out of 9124 total) in the "shoot/meristem" library, representing 0.44 actin ESTs for every 1000 total ESTs. Comparing this value with its expression levels from other libraries gives an indication of how actin transcript levels vary relative to total transcript populations.

\section{Calculation of Fold Ranges and Transcript Variation}

We used "fold statistics" to determine the variation of relative expression levels among the different libraries, which involved calculating the percentage of libraries that had relative expression levels (for a given transcript) within some range (2-fold, 3 -fold, etc.). Because the distribution of EST data is approximated by a Poisson distribution $(7,19)$, it was appropriate to calculate the ranges using a square root transformation based on the following equations:

$$
\begin{array}{ll}
\text { Lower range }=\mathrm{E} \cdot 1 / \sqrt{\mathrm{F}} & {[\text { Eq. 1] }} \\
\text { Upper range }=\mathrm{E} \cdot \sqrt{\mathrm{F}} & {[\text { Eq. 2] }}
\end{array}
$$

where $\mathrm{E}$ is the average relative expression level for a given transcript from 
the various cDNA libraries sampled and $F$ is the fold difference. For example, to determine how many libraries contained transcript $\mathrm{X}$ within a 3 -fold level of expression ( $\mathrm{F}=3$ ), if the average relative expression level for transcript $X$ were $0.1(E=0.1)$, the range would be calculated as 0.058 to 0.173 (a 3-fold difference). We then found the percentage of libraries that fell into each range for each transcript and examined the resulting plots. We also ranked transcripts using the median relative expression level rather than the average (see Equations 1 and 2) and arrived at similar results. Within our data set, calculated ranges led to a symmetrical distribution (an approximately equal number of libraries falling into a range above and below the average) across a 10 -fold range.

\section{RESULTS AND DISCUSSION}

Figure 1 shows transcripts whose relative expression levels fell into fold ranges when all 27 tomato libraries were considered together. The lines toward the top of Figure 1 represent transcripts that tend to be expressed at constant levels in many different tissues, where a "perfect" candidate transcript would be present in $100 \%$ of the libraries at less than a 2-fold range of expression. Heat shock cognate protein 80 (acc. no. TC123867) was present in $89 \%$ of libraries within a 10 -fold range, while the highest-ranked within a 3fold range included DnaJ-like protein (acc. no. TC124053) in 60\% of libraries, translationally controlled tumor protein (TCTP; acc. no. TC115845), two $\alpha$-tubulins (acc. nos. TC115716 and TC115782), cyclophilin (acc. no. TC115937), and GAPDH (acc. no. TC115908). In general, ranking at any of the fold ranges gives similar results (i.e., the slopes of the lines are similar).

It is expected that fold differences will be greater in EST data than in data from direct measurement [i.e., hybridization or reverse transcription PCR (RT-PCR)]. Random variation is high in EST analyses since the number of ESTs representing most genes is usually small, making the data inherently noisy $(20,21)$. Even so, Figure 1

Table 2. Highest-Ranking Housekeeping Control Genes in Various Tomato Plant Tissues

\begin{tabular}{|c|c|c|c|c|c|}
\hline & Putative Gene & Acc. No. & $\begin{array}{l}\text { Tissue-Specific } \\
\text { Expression Level }\end{array}$ & $\begin{array}{l}\text { 2-Fold } \\
(\%)\end{array}$ & $\begin{array}{l}\text { 3-Fold } \\
\text { (\%) }\end{array}$ \\
\hline \multirow[t]{14}{*}{ Leaves } & GAPDH (plastidic) & TC123860 & 4.36 & 100 & 100 \\
\hline & GAPDH (plastidic) & TC124167 & 1.79 & 100 & 100 \\
\hline & GAPDH (non-phosph. cytosolic) & TC124579 & 0.20 & 100 & 100 \\
\hline & Phosphoglycerate kinase & TC123837 & 1.11 & 100 & 100 \\
\hline & Phosphoglycerate kinase & TC116028 & 0.66 & 100 & 100 \\
\hline & Catalase & TC115751 & 1.27 & 100 & 100 \\
\hline & Catalase & TC115865 & 0.98 & 100 & 100 \\
\hline & Cys protease & TC124125 & 0.69 & 100 & 100 \\
\hline & Cys protease & TC116356 & 0.20 & 100 & 100 \\
\hline & $\alpha$-Tubulin & TC115716 & 1.10 & 100 & 100 \\
\hline & Ubiquitin & TC115896 & 0.53 & 100 & 100 \\
\hline & Actin & TC124219 & 0.42 & 100 & 100 \\
\hline & DnaJ-like protein & TC124053 & 0.46 & 100 & 100 \\
\hline & Translation initiation factor $5 \mathrm{~A}-4$ & TC116211 & 1.02 & 100 & 100 \\
\hline \multirow[t]{5}{*}{ Roots } & $60 S$ acidic ribosomal protein & TC116351 & 0.33 & 100 & 100 \\
\hline & Phosphoglycerate kinase & TC116028 & 1.38 & 100 & 100 \\
\hline & Ubiquitin & TC116485 & 0.48 & 75 & 100 \\
\hline & UDP-glucose:protein transglucosylase & TC124606 & 0.97 & 50 & 100 \\
\hline & $\alpha$-Tubulin & TC115716 & 2.44 & 50 & 75 \\
\hline \multirow[t]{5}{*}{ Fruits } & Phosphoglycerate kinase & TC123837 & 0.22 & 100 & 100 \\
\hline & Chaperonin- 60 beta chain prec. & TC115713 & 2.06 & 100 & 100 \\
\hline & DnaJ-like protein & TC123959 & 0.22 & 100 & 100 \\
\hline & DnaJ-like protein & TC124053 & 0.73 & 100 & 100 \\
\hline & AT5g28840/F7P1_20 & TC123964 & 0.41 & 75 & 100 \\
\hline \multirow[t]{5}{*}{ Flowers } & $\alpha$-Tubulin & TC115716 & 1.12 & 100 & 100 \\
\hline & Heat shock cognate protein 80 & TC123867 & 3.34 & 100 & 100 \\
\hline & DnaJ-like protein & TC124053 & 0.99 & 100 & 100 \\
\hline & Phosphoglycerate kinase & TC123837 & 0.60 & 80 & 100 \\
\hline & GAPDH (cytosolic) & TC115908 & 1.75 & 80 & 80 \\
\hline \multicolumn{6}{|c|}{$\begin{array}{l}\text { Fold percentages show the percentage of cDNA libraries ( } n=3,4,4 \text {, and } 5 \text { for } \\
\text { leaves, roots, fruits, and flowers, respectively) with relative expression levels for } \\
\text { a given gene within a 2- or 3-fold range. Relative expression levels are in units of } \\
\text { ESTs per } 1000 \text { total ESTs. }\end{array}$} \\
\hline
\end{tabular}

suggests the impossibility of having a single housekeeping control gene for all tissues, since no transcript was expressed in more than $89 \%$ of libraries within a 10 -fold range.

When the data were analyzed by organ, candidate housekeeping control genes emerged (Table 2). For each organ, at least two transcripts were found that occurred within a 2 -fold range of expression in all respective libraries. $\alpha$-Tubulin (acc. no. TC115716) was found at constant levels in leaves, roots, and flowers, whereas DnaJ-like protein (acc. no. TC124053) and phosphoglycerate kinase (acc. no. TC123837) were constant in leaves, fruits, and flowers (Table 2). A small family of DnaJ- like proteins (acc. nos. TC123959, TC123960, and TC124053) were somewhat constant in all organs. Only phosphoglycerate kinase was among the highest ranked for all organs. It must be noted, though, that of the two distinct phosphoglycerate kinase transcripts, one (acc no. TC116028) appears more constantly expressed in roots, and the other (acc. no. TC123837) in fruits and flowers. As expected, most highly ranked transcripts had an abundance of ESTs. The notable exception was $60 \mathrm{~S}$ acidic ribosomal protein (acc. no. TC116351) in roots, which had only one EST in each library, making it a possible false positive.

Interestingly, some of the highest- 


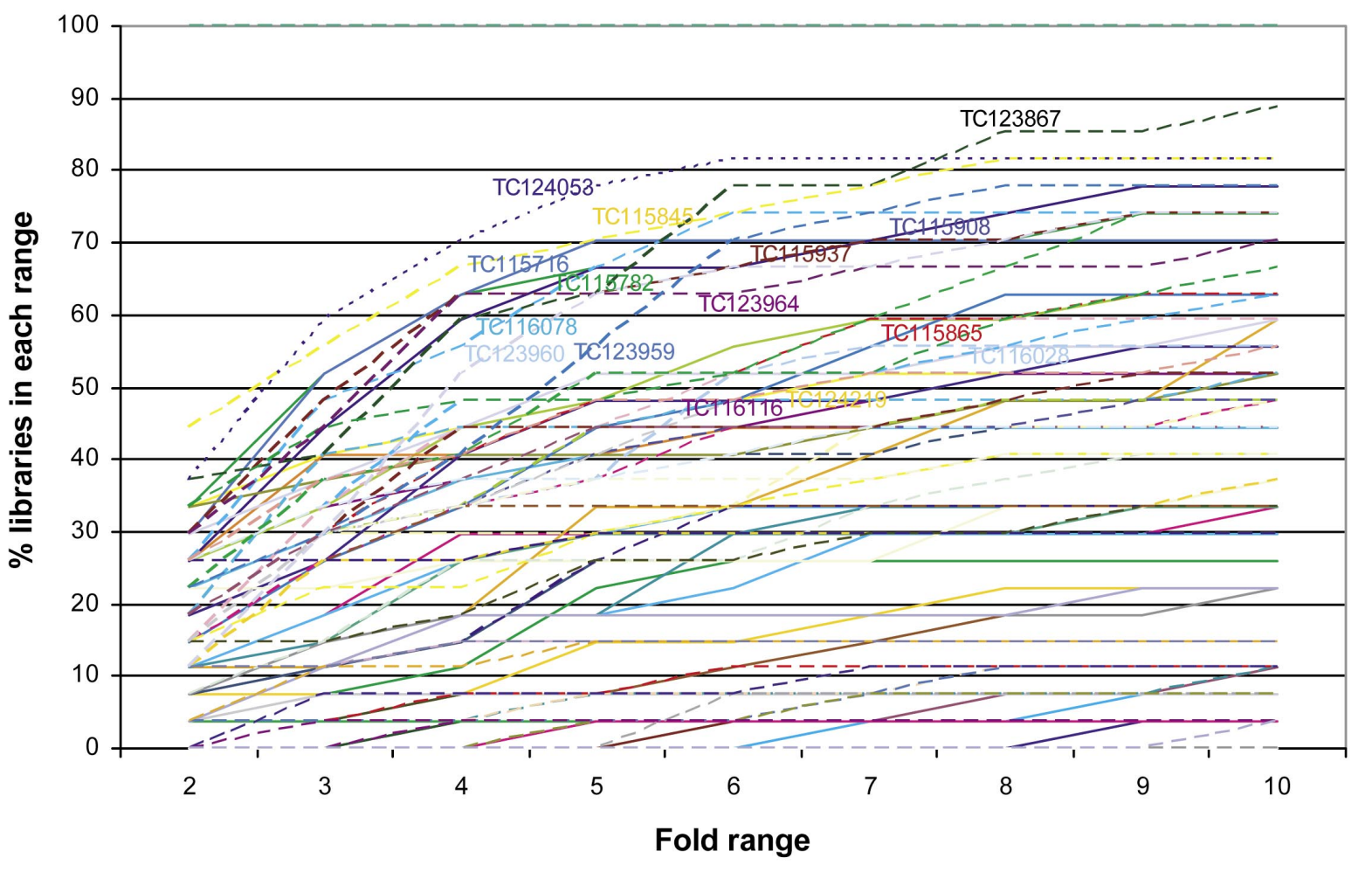

Figure 1. Percentage of tomato cDNA libraries $(n=27)$ that contain expressed sequence tags $(E S T s)$ for given genes within various fold ranges of relative expression. Each line represents a tentative consensus sequence (TC) from the TIGR TGI. For example, the relative expression level of a DnaJ-like protein was within a 3-fold range in 59\% of libraries. Putative identifications for labeled accessions are as follows: TCTP (TC115845), $\alpha$-tubulin (TC115716 and TC115782), actin (TC124219 and TC116116), heat shock cognate protein 80 (TC123867), phosphoglycerate kinase (TC116028), DnaJ-like protein (TC123959, TC123960, and TC124053), GAPDH (acc. no. TC115908), translation elongation factor EF-2 (TC116078), unknown protein AT5g28840/F7P1_20 (TC123964), catalase (TC115865), cyclophilin (TC115937).

ranking transcripts in individual organs (Table 2) ranked very low when all tissues were considered together (Figure 1), and vice versa. For example, although $100 \%$ of leaf, fruit, and flower libraries contained phosphoglycerate kinase (acc. no. TC123837) within a 2-fold range of expression (Table 2), when all libraries were considered together only $15 \%$ contained the transcript within a 2 -fold range. Similarly, translationally controlled tumor protein (TCTP; acc. no. TC115845) was among the highest-ranked transcripts when all libraries were considered together (Figure 1) but was not when organs were considered separately. This suggests that some transcripts are more appropriate controls for RNA experiments within organs, whereas others may be better suited for experiments between organs.

It is sometimes useful for house- keeping controls in an mRNA experiment to have expression levels similar to those of the other genes being tested. Relative expression levels from EST data can be used to pick candidate control genes with appropriate mRNA levels (Table 2). For example, to measure the differential expression of a gene in leaves with an expression level of 0.60 ESTs per 1000 total ESTs, candidate housekeeping controls include ubiquitin (acc. no. TC115896), phosphoglycerate kinase (acc. no. TC116028), and cys protease (acc. no. TC124125), which have expression levels around 0.60 (Table 2). Similarly, comparing expression levels in different tissues allows the selection of appropriate housekeeping controls. For example, $\alpha$-tubulin (acc. no. TC115716) is a candidate control in an experiment involving both leaf and flower tissue, since its relative expression level is identical in these tissues (Table 2).

This analysis provided no evidence that the most frequently used housekeeping controls, actin and GAPDH, are better than other candidate control transcripts. Actin transcripts appeared just once among the highest-ranked controls in individual organs (Table 2) and were present in only $52 \%$ of all libraries within a 10 -fold range (Figure 1). This could be partly due to individual actin transcripts having a smaller number of ESTs than others such as $\alpha$-tubulin. These data emphasize that the methods we used can identify potential housekeeping controls but cannot always prove that lower abundance ESTs are not also appropriate housekeeping controls. Examining EST data from several related species to increase sample size would allow the identification of lower abundance 
housekeeping controls. Nevertheless, our analysis made it clear that 2 (acc. no. TC124219 and TC116116) of the 15 putative actin transcripts appear more constant than the other 13 (data not shown). Putative GAPDH transcripts were among the highestranked in leaves and flowers (Table 2) and were present in $78 \%$ of all libraries within a 10-fold range (Figure 1). However, the four GAPDH transcripts shown in Table 2 differ significantly from each other ( $<75 \%$ identity), and none were among the highest-ranked transcripts in multiple organs. Transcript levels of plastid GAPDH (acc. no. TC123860 and TC124167) and nonphosphorylating cytosolic GAPDH (acc. nos. TC124579) were constantly expressed in leaves, whereas another cytosolic GAPDH (acc. no. TC115908) was constant in flowers.

Although we identify candidate housekeeping controls, it is important to note that none of those used to date have proven adequate in all situations $(9,12)$. Therefore, it would not be valid to choose the "best" housekeeping control and then use that control indiscriminately, even within a given organ. Because mRNA levels even of housekeeping genes can vary, it is always preferable to choose housekeeping controls based on previous experimental evidence in a context-dependent fashion. The analysis here identifies transcripts, and methods for finding them, that would be logical starting points when mRNA housekeeping controls are needed. This analysis could be performed for other tissues/species for which significant EST data exist, and similar analyses could be applied to other types of expression databases such as those for the serial analysis of gene expression (SAGE).

\section{ACKNOWLEDGMENTS}

The cDNA libraries in the TIGR TGI were constructed in the laboratories of Steve Tanksley, Greg Martin, and Jim Giovannoni. We thank Debra Coker for computer expertise, Sophia Clotho for her advice, and reviewers for helpful comments (especially the suggestion of using a square root transformation in Equations 1 and 2).

\section{REFERENCES}

1.Cooke, R., M. Raynal, M. Laudié, and M. Delseny. 1997. Identification of members of gene families in Arabidopsis thaliana by contig construction from partial cDNA sequences: 106 genes encoding 50 cytoplasmic ribosomal proteins. Plant J. 11:1127-1140.

2.Barakat, A., K. Szick-Miranda, I.-F. Chang, R. Guyot, G. Blanc, R. Cooke, M. Delseny, and J. Bailey-Serres. 2001. The organization of cytoplasmic ribosomal protein genes in the Arabidopsis genome. Plant Physiol. 127: 398-415.

3.Adams, M.D., R.D. Kerlavage, R.A. Fleischmann, C.J. Fuldner, N.H. Bult, E.F. Lee, K.G. Kirkness, J.D. Weinstock, et al. 1995. Initial assessment of human gene diversity and expression patterns based upon 83 million nucleotides of cDNA sequence. Nature 377:3-17.

4.Coker, J.S., D. Jones, and E. Davies. 2003. Identification, conservation, and relative expression of V-ATPase cDNAs in tomato plants. Plant Mol. Biol. Rep. 21:145-158.

5.Audic, S. and J.-M. Claverie. 1997. The significance of digital gene expression profiles. Genome Res. 7:986-995.

6.Greller, L.D., and F.L. Tobin. 1999. Detecting selective expression of genes and proteins. Genome Res. 9:282-296.

7.Stekel, D.J., Y. Git, and F. Falciani. 2000. The comparison of gene expression from multiple cDNA libraries. Genome Res. 10: 2055-2061.

8.Blake, J.B., M. Kaern, C.R. Cantor, and J.J. Collins. 2003. Noise in eukaryotic gene expression. Nature 422:633-637.

9.Ivell, R. 1998. A question of faith-or the philosophy of RNA controls. J. Endocrinol. 159:197-200.

10.Thellin, O., W. Zorzi, B. Lakaye, B. De Borman, B. Coumans, G. Hennen, T. Grisar, A. Igout, and E. Heinen. 1999. Housekeeping genes as internal standards: use and limits. J. Biotechnol. 75:291-295.

11.Suzuki, T., P.J. Higgins, and D.R. Crawford. 2000. Control selection for RNA quantitation. BioTechniques 29:332-337.

12.Lee, P.D., R. Sladek, C.M.T. Greenwood, and T.J. Hudson. 2001. Control genes and variability: absence of ubiquitous reference transcripts in diverse mammalian expression studies. Genome Res. 12:292-297.

13.Van der Hoeven, R., C. Ronning, J. Giovannoni, G. Martin, and S. Tanksley. 2002. Deductions about number, organization, and evolution of genes in the tomato genome based on analysis of a large expressed sequence tag collection and selective genomic sequencing. Plant Cell 14:1441-1456.

14.Rounsley, S.D., A. Glodek, G. Sutton, M.D. Adams, C.R. Somerville, J.C. Venter, and A.R. Kerlavage. 1996. The construction of Arabidopsis expressed sequence tag assemblies: a new resource to facilitate gene identification. Plant Physiol. 112:1177-1183.

15.White, J.A., J. Todd, T. Newman, N. Focks, T. Girke, O.M. de Ilarduya, J.G. Jaworski, J.B. Ohlrogge, and C. Benning. 2000. A new set of Arabidopsis expressed sequence tags from developing seeds. The metabolic pathway from carbohydrates to seed oil. Plant Physiol. 124:1582-94.

16.Lamblin, A.F., J.A. Crow, J.E. Johnson, K.A. Silverstein, T.M. Kunau, A. Kilian, D. Benz, M. Stromvik, et al. 2003. MtDB: a database for personalized data mining of the model legume Medicago truncata transcriptome. Nucleic Acids Res. 31:196-201.

17.Ronning, C.M., S.S. Stegalkina, R.A. Ascenzi, O. Bougri, A.L. Hart, T.R. Utterbach, S.E. Vanaken, S.B. Riedmuller, et al. 2003. Comparative analyses of potato expressed sequence tag libraries. Plant Physiol. 131: 419-429.

18.Quackenbush, J., J. Cho, D. Lee, F. Liang, I. Holt, S. Karamycheva, B. Parvizi, G. Pertea, R. Sultana, and J. White. 2001. The TIGR Gene Indices: analysis of gene transcript sequences in highly sampled eukaryotic species. Nucleic Acids Res. 29:159-164.

19.Romualdi, C., S. Bortoluzzi, and G.A. Danieli. 2001. Detecting differentially expressed genes in multiple tag sampling experiments: comparative evaluation of statistical tests. Hum. Mol. Genet. 10:2133-2141.

20.Hillier, L.G., M. Lennon, M. Becker, B. Bonaldo, N. Chiapelli, T. Dietrich, A. DuBuque, W. Favello, and M. Gish. 1996 Generation and analysis of 280,000 human expressed sequence tags. Genome Res. 6: 807-828.

21.Wolfsberg, T., and D. Landsman. 1997. A comparison of expressed sequence tags (ESTs) to human genomic sequences. Nucleic Acids Res. 25:1626-1632.

Received 15 May 2003; accepted 17 June 2003.

Address correspondence to Jeffrey $S$. Coker, Department of Botany, North Carolina State University, Campus Box 7612, Raleigh, NC 27695, USA. e-mail: jeffreycoker@hotmail.com 